\title{
ACTIVIDADES 2014
}

XXVIII Congreso Centroamericano de

Psiquiatría

18 al 22 de junio

Punta Cana, República Dominicana

International Congress of Cognitive

Psychotherapy

24 al 27 de junio

Hong Kong, China

International Congress of the Royal

College of Psychiatrists

24 al 27 de junio

Londres, Reino Unido

II Congreso Internacional de Ciencias de la Educación y del Desarrollo

25 al 27 de junio

Granada, España

IV Congreso Internacional de Docencia

Universitaria

2 al 4 julio

Tarragona, España

9th Conference of the International Test

Commission

2 al 5 de julio

San Sebastián, España

Congreso Internacional Multidisciplinar de Investigación Educativa

3 al 4 julio

Segovia, España

$28^{\circ}$ Congreso Internacional de Psicología

Aplicada "De la crisis al bienestar sustentable" (IAAP)

8 al 13 de julio

Paris, Francia

22nd. International Congress of the International Association for Cross

Cultural Psychology

15 al 19 de julio

Reims, Francia
Neurology and Psychiatry for Primary Care

18 al 20 de julio

Carolina del Sur, USA

V Congreso Regional de la Sociedad Interamericana de Psicología (SIP)

"Para una Psicología comprometida y con relevancia social"

21 al 24 de julio

San Salvador, El Salvador

Conferencia Internacional sobre Conocimiento, Innovación y Empresa 22 al 25 de julio

Riga, Letonia

Convención Anual División 9 APA, SPSSI

"Desigualdades, injusticias e intersecciones: Quién, cuándo, por qué, cómo.... y qué puede hacer la Psicología" 7 al 10 de agosto

Washington, USA

VIII Congreso de la Asociación Psicoanalítica del Uruguay y XVIII Jornadas de Psicoanálisis: Sexualidad una búsqueda sin fin

15 al 16 de agosto

Montevideo, Uruguay

$2^{\circ}$ Congreso Ibero Latinoamericano y 3er. Encuentro Ibero Latinoamericano de Grupos de Investigación en Psicología Política

19 al 23 de agosto

San Luis Potosí, Mexico

National Conference on Addiction

Disorders

22 al 26 de agosto

St. Louis, USA.

7th World Congress for Psycotherapy

25 al 29 de agosto

Durban, Sudáfrica 
3er. Congreso Internacional de Psicología del Desarrollo

27 al 29 de agosto

Aguascalientes, México

1er. Congreso Universitario de Psicología Clínica

28 de agosto

Montevideo, Uruguay

III Seminario de Salud y Seguridad en el Trabajo

29 al 30 de agosto

Cartagena, Colombia

5a. Conferencia Internacional de Psicología Comunitaria

3 al 6 de setiembre

Fortaleza-Ceará, Brasil.

IX Congreso Ibero-Americano de

Psicología

10 al 13 de septiembre

Lisboa, Portugal

XIV Congreso Internacional Grupo, Psicoterapia y Psicoanálisis

9 al 11 de octubre

Avilés, Asturias

Segundo Congreso de las Américas sobre Factores Psicosociales, Estrés y Salud Mental en el Trabajo

14 al 17 de octubre

Ciudad de México, México

I Congreso Latinoamericano para el Avance de la Ciencia Psicológica

15 al 18 de octubre

Buenos Aires, Argentina
VIII Seminario Internacional de Psicología de la Salud en Atención Primaria

10 al 14 de noviembre

La Habana, Cuba

\section{Congreso Internacional de Psicología} Clínica

14 al 16 de noviembre

Sevilla, España

III Congreso Iberoamericano de Psicología de la Salud

14 al 16 de noviembre

Sevilla, España

1er Congreso uruguayo de

Acompañamiento Terapéutico

20 al 22 de noviembre

Montevideo, Uruguay

Congreso Internacional "Infancia en

contextos de riesgo"

20 al 22 de noviembre

Huelva, España

Congreso Internacional de Salud y Ergonomía Ocupacional CISEO 2014

26 al 28 de noviembre

Lima, Perú

VI Congreso Internacional de Investigación y Práctica Profesional en Psicología.

Décimo Encuentro de Investigadores en

Psicología del Mercosur y XXI Jornadas de Investigación

22 al 29 de noviembre

Buenos Aires, Argentina 